\title{
Recruiting and Retaining Low-Income Latinos in Psychotherapy Research
}

\author{
Jeanne Miranda \\ Department of Psychiatry Georgetown University Medical Center
}

Francisca Azocar

Department of psychology University of California, San Francisco, and San Francisco General Hospital

Kurt C. Organista

School of Social Welfare University of California, Berkeley

Ricardo F. Muñoz

Department of psychology University of California, San Francisco, and San Francisco General Hospital

\section{Alicia Lieberman}

Department of psychology University of California, San Francisco, and San Francisco General Hospital

\section{ABSTRACT}

This article offers suggestions for recruiting and retaining low-income Latinos in treatment studies. Because Latinos underuse traditional mental health services, places such as medical centers or churches with large Latino constituents are suggested as useful alternative sources. To keep Latinos in research protocols, providing culturally sensitive treatments are necessary. Culturally sensitive treatments should incorporate families as part of recruitment efforts, particularly older men in the family. In addition, showing respect is an important aspect of traditional Latino culture that includes using formal titles and taking time to listen carefully. Finally, traditional Latinos tend to like interactions with others that are more warm and personal than is generally part of a research atmosphere.

Correspondence may be addressed to Jeanne Miranda, Department of Psychiatry, Georgetown University Medical Center, 3800 Reservoir Road Northwest, Washington, DC, 20007-2197.

Received: July 7, 1995

Revised: October 19, 1995

Accepted: December 1, 1995

Are Latinos more likely to respond to psychotherapy or pharmacotherapy for treatment of an episode of depression? Are Latinos more likely to drop out of cognitive-behavioral therapy or interpersonal psychotherapy? Are recent immigrants from Mexico more likely to respond to a highly structured psychotherapy or a less structured treatment? All of these questions are important for serving the mental

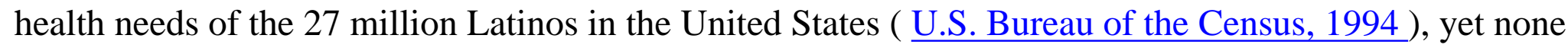


can be answered with currently available data from treatment outcome studies.

Psychotherapy studies examining Latino populations have been extremely limited in scope. A recent meta-analytic review of all psychotherapy studies conducted in the United States with Latino participants in the 20-year period starting in 1970 identified 135 such studies ( Navarro, 1993). Of the 123 studies that could be located by the reviewer, 28 were single-case reports, 27 were single-group, preassessment-postassessment (pre-post) comparisons, and 48 were nonexperimental designs, included no quantitative data, or were primarily theoretical in nature. The remaining 20 reports described 15 unique studies with experimental or quasi-experimental designs. Of these, 11 were studies of children or adolescents with behavioral or drug problems, and 4 were studies with adults. Of these 4,1 did not use randomized assignment, and the other 3 focused on self-control of aggressive behavior toward children in Puerto Rican fathers ( Figueroa-Torres \& Pearson, 1979), assertiveness training in mainland Puerto Rican women ( Comas-Diaz \& Duncan, 1985), and cognitive-behavioral therapy for depression with Puerto Rican women ( Comas-Diaz, 1981a, 1981b).

Psychotherapy studies of the general population rarely include Latinos. For example, the National Institute of Mental Health Treatment of Depression Collaborative Research Program ( Elkin et al., 1989 ), an extremely important psychotherapy outcome study, included only $11 \%$ ethnic minorities. Furthermore, only $2 \%$ were Latinos. Six additional studies have compared psychotherapy with pharmacotherapy for treatment of depression. One of these reports did not mention ethnic or racial characteristics of participants ( Blackburn, Bishop, Glen, Whalley, \& Christie, 1981); one reported that $17 \%$ of the participants were African American ( Murphy, Simons, Wetzel, \& Lustman, 1984 ); and four reported that $10 \%$ or less of the participants were not White, with no reference to Latinos ( DiMascio et al., 1979; Hersen, Bellack, Himmelhoch, \& Thase, 1984 ; Hollon et al., 1992 ; and Rush, Beck, Kovacs, \& Hollon, 1977). We are aware of only one psychotherapy outcome study examining treatment of depression that included a Spanish-speaking sample (Comas-Diaz, 1981a, 1981b). Clearly, the failure to recruit Latinos in psychotherapy studies limits our understanding of the effectiveness of interventions with this population.

Several important research efforts have evaluated treatments that address the unique difficulties associated with immigration for Latinos. For example, cuento therapy, a treatment that provides ethnically appropriate role models for Puerto Rican children and adolescents to bridge bicultural conflict, has been shown to be effective ( Costantino, Malgady, \& Rogler, 1986; Malgady, Rogler, \& Costantino, $\underline{1990}$ ), as has bicultural effectiveness training aimed at treating cultural conflict and acculturative stress in immigrant families ( Szapocznik et al., 1986). Latinos do face special stresses associated with immigration, and appropriate interventions for those stresses are needed. However, this population also experiences typical disorders such as depression and anxiety; these disorders are known to respond to standardized treatments. By focusing on recruiting and retaining Latinos in traditional psychotherapy studies, the overall effectiveness of different psychotherapies can be evaluated with Latinos. In addition, findings from this research could identify types of treatments or components of treatments that require more specific cultural adaptation. Finally, by including diverse populations in psychotherapy research, 
important questions regarding the interaction of environmental background and treatment can be answered. We strongly recommend that representative samples of Latinos be included in efficacy trials of psychotherapy. Certainly, Latinos must be evaluated in effectiveness research examining the applicability of treatments to the general population. The purpose of this article is to provide information for researchers regarding recruiting and retaining Latinos in psychotherapy trials.

In this article, we discuss identification of Latino populations with mental disorders. Then, we discuss some of the basics of Latino culture to help readers understand how to modify typical research protocols to become more culturally sensitive to Latinos. Finally, we discuss a series of intervention studies that we have conducted to illustrate useful strategies for recruiting and retaining Latinos.

\section{Access to Latinos in the United States}

Are there enough Latinos to recruit into psychotherapy trials? Latinos made up $10.2 \%$ of the United States population in 1995 and will be $11.3 \%$ by the year 2000 ( U.S. Bureau of the Census, 1994 ). Therefore, they are now and will increasingly become important subgroups of psychotherapy study participants. In certain metropolitan areas, Latinos are now such a large proportion of the population that it makes no sense to exclude them from clinical trials. For example, in El Paso, Texas, they are $70 \%$ of the population; in Albuquerque, New Mexico, 37\%; and in the Los Angeles Riverside-Orange County area, 33\% ( U.S. Bureau of the Census, 1994 ).

Latinos are a diverse group of individuals with roots in the countries of Latin America, many of whom speak Spanish, and most of whom carry the blended cultural and racial heritage of the Spanish colonists and of the indigenous peoples of the Americas. Latinos cluster in Miami, New York City, Los Angeles, and Texas, but are increasingly visible in other areas as well. For example, Latinos now number more than 225,000 in the metropolitan Washington, D.C., area ( U.S. Census Bureau, 1990), and they make up $11 \%$ of the population in the Chicago metropolitan area.

Latinos span every racial group and come from Mexico (63\%), Cuba (5\%), Puerto Rico (11\%), Central and South America (14\%), as well as other countries (7\%). Political and economic factors often influence immigration patterns and, therefore, socioeconomic status of Latinos in the United States. Overall, Latinos have lower incomes than do non-Hispanic Whites; Cubans are the most affluent and educated of Latinos in the United States, followed by Central and South Americans, Mexicans, and Puerto Ricans. In terms of poverty, only about $10 \%$ of non-Latino Whites live below the poverty level, compared with $39 \%$ of Puerto Ricans, 30\% of Mexican Americans, and 18\% of Cuban Americans (

Not only must psychotherapy studies have sufficient numbers of Latinos, or a subgroup of Latinos, to recruit, but a sufficient number of Latinos with mental disorders must be available to include them in outcomes research. Early epidemiological studies of Latinos found that rates of disorder vary by subgroup ( Moscicki, Locke, Rae, \& Boyd, 1989 ; Narrow, Rae, Moscicki, Locke, \& Regier, 1990 ), with new immigrants tending to have lower rates of disorder. 
The newest contribution to understanding the epidemiology of mental disorders in Latinos is the National Comorbidity Survey ( Kessler et al., 1994 ). This study surveyed individuals 15 to 54 years old who were representative of the noninstitutionalized U.S. population as a whole, including Latinos. Latinos were found to have a higher prevalence of current affective disorders and active comorbidity (three or more) disorders than non-Latino Whites. There were no disorders with lower prevalence rates for Latinos than those for Whites. This survey provides the strongest evidence to date of significant risk for mental disorders in Latinos. This result is not surprising, given the strong inverse relationship between socioeconomic status and mental disorders that has been consistently found in epidemiological studies and the high rates of poverty of Latinos residing in the United States.

\section{Use of Services by Latinos}

If Latinos with mental disorders are prevalent, why are they missing from psychotherapy outcome studies? Part of the answer comes from recruitment strategies for typical studies. Psychotherapy studies generally recruit from psychiatric outpatient facilities. Several investigations have found that poor and

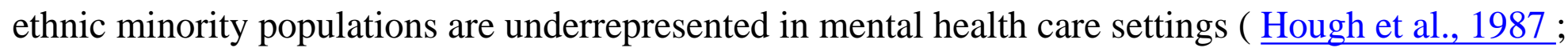
Loo, Tong, \& True, 1989; Meinhardt \& Vega, 1987; Snowden \& Cheung, 1990; Vernon \& Roberts, 1982 ; Wells, Hough, Golding, Burnam, \& Karno, 1987 ). Latinos, in particular, demonstrate a clear pattern of underutilization of mental health services ( Sue, Fujino, Hu, Takeuchi, \& Zane, 1991 ), with immigrants and those less acculturated having extremely low rates of service use ( Wells et al., 1987).

Given that Latinos are seriously underrepresented in mental health settings, what factors may keep them from participating in mental health services and psychotherapy research? Unfortunately, many theories that are used to explain persisting differences in mental health service use between Latinos and Whites have not been empirically supported. For example, negative attitudes toward mental health services were hypothesized as factors keeping Latinos from treatment. However, many studies find that Latinos hold positive views of mental health services and mental health professionals, often even more positive than views held by Whites ( Acosta \& Sheehan, 1976a , 1976b; Karno \& Edgerton, 1969; Leaf, Bruce, Tischler, \& Holzer, 1987). Similarly, the theory that Latinos underutilize formal services relative to Whites because of their greater use of traditional healers or family members as sources of help has not

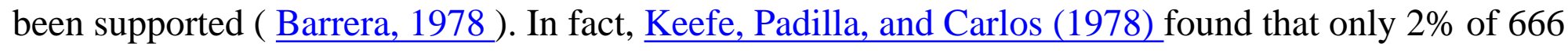
Mexican Americans surveyed in Los Angeles had consulted a curandero, who dispenses herbal medicines and potions, for emotional problems in the previous year. Of course, use of traditional healers may be underreported because of social desireability ( Comas-Diaz, 1981a , 1981b; De La Cancela \& Zavala, 1983 ). Regardless of absolute accuracy of reports, use of traditional healers should only account for a small portion of underutilization of mental health services by Latinos.

Another hypothesis has been that Latinos use nonpsychiatric physicians rather than mental health professionals for psychological problems ( Barrera, 1978; Edgerton, Karno, \& Fernandez, 1970; Karno

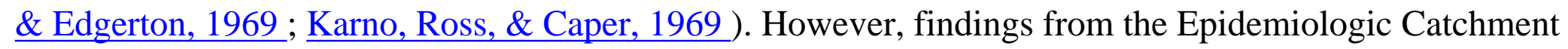


Area (ECA) studies did not support this speculation. Among those who had a diagnosable mental disorder, Mexican Americans were as likely as non-Hispanic Whites to see a mental health specialist when they did make a mental health visit ( Hough et al., 1987); however, they were only half as likely to make a mental health visit (22\% for non-Hispanic Whites vs. 11\% for Mexican Americans).

Availability and accessibility to mental health services are clearly a factor in Latinos' general underutilization of services. Specifically, ethnic minority and low-socioeconomic-status individuals report more instrumental barriers to mental health care, such as lack of insurance, time, child care, and transportation than do White and higher income individuals ( Leaf et al., 1987; Marin, Marin, Padilla, \& de la Rocha, 1983; Takeuchi, Leaf, \& Kuo, 1988 ). Removing such barriers clearly improves utilization. For example, Norquist and Wells (1991a , 1991b) found that a Mexican American sample with Medi-Cal insurance used more services than did those without insurance. Similarly, providing services in Spanish is essential for recruiting monolingual Spanish-speaking Latinos. Thus, providing access to treatment is essential for recruiting and retaining Latinos in psychotherapy studies.

\section{Cultural Factors Influencing Latinos' Use of Services}

Latinos may fail to participate in psychotherapy studies because of cultural factors. Developing culturally sensitive strategies for recruiting and retaining Latinos in treatment outcome studies may improve access to this population as well. Although Latinos are a heterogenous group and cultural stereotyping is not appropriate, certain commonalities among Latinos can guide efforts to recruit and retain them in treatment.

In general, Latinos have strong and traditional family values, commonly known as familísmo ( Marín \& Marín, 1991 ). The family, characterized as including both nuclear and extended family members, is central to and more important than the individual. Families are held together by strong bonds of loyalty and commitment ( Bernal \& Gutierrez, 1988 ). Machísmo and marínismo are important aspects of the hierarchical nature of more traditional family units. Machísmo refers to the father as the most powerful member in the family, who makes the major decisions, provides both financial and emotional stability and protects the family from any danger. Marianísmo, "the long-suffering mother," refers to the spiritual superiority of the mother, capable of enduring any suffering and self-sacrifice to help the husband and children ( Bernal \& Gutierrez, 1988). To recruit and retain Latinos in psychotherapy studies, the family structure should be considered. For example, a Latina in one of our studies was unable to participate in treatment because her husband disapproved of her attending our sessions. Responding to her request to do so, we discussed the treatment with him directly; he then agreed to allow her to participate. In many cases, the "man of the family" may need to be included in discussions before his wife is recruited into treatment.

Respect toward male figures, as well as older adults in general, are also important aspects of Latino culture. Personalísmo refers to the dignified approach to personal associations that many Latinos prefer. Important aspects of this personal approach include the use of formal language (Usted, Señor, Señor, 
etc.), as well as formal greetings, in professional relationships. Traditional Latinos show great respect to professionals. Along with showing respect, Latinos are expected to be warm and personal in their interactions with others, a concept known as simpatía ( Marín \& Marín, 1991 ). In particular, Latinos tend to appreciate and respond well to the simpatía of others. Traditional approaches to recruiting participants into treatment outcome studies may be perceived as both too informal or too cold for many Latinos. Studies successful at recruiting and retaining Latinos (e.g., Rogler, Cortes, \& Malgady, 1991; Muñoz \& Ying, 1993 ) have used research staff who treat older Latinos with respect, using formal titles, while being warm and personable. We believe the ability to use the polite form of the word "you" ("usted," plus the related verb forms) allows for a simultaneous expression of respect and of warm closeness that is difficult to do in English. Other touches, however, that convey the importance of the person and his or her family (e.g., remembering the names of their children and asking about them during each interview) may be extremely helpful in developing the relationship necessary to engage Latinos in psychotherapy research.

Many Latinos are Catholic and hold a holistic view of the self, integrating the body, mind, and soul. Religion is supplemented with folk beliefs to explain as well as solve problems. Some folk beliefs encourage use of faith healers. For example, espiritismo is a faith healing system from Puerto Rico that uses a medium to exorcise spirits causing the problem. Cuban santería includes potions, herbs, and candles to extract problems caused by natural and supernatural causes. Curanderos are commonly used throughout Latin America. Folk beliefs explain psychiatric symptomatology as the result of nervios (being too nervous), susto (magical fright) and mal de ojo (hex).

Although more traditional Latinos are more likely to use folk healers, actual use in the United States is quite low ( Barrera, 1978). However, remedios caseros (home remedies) are often used. Although these alternative treatments and folk beliefs about psychiatric symptoms are unlikely to interfere with Latinos' participation in research, these issues may affect diagnoses and exclusionary criteria. For example, Latinos may present with culturally appropriate beliefs that would be considered psychotic out of context. Many of our patients have reported talking with deceased relatives, seeing religious visions, and worrying about hexes; these can be culturally appropriate and not representative of psychotic processes. Similarly, Latinos may present with additional symptoms that are explained culturally, such as ataques de nervios (attacks of nerves; Guarnaccia, Canino, Rubio-Stipec, \& Bravo, 1993 ).

In summary, both organizational factors (i.e., access to affordable treatment, treatment available in Spanish, etc.) as well as cultural factors (i.e., services that are congruent with Latino family structure, that are warm and personal, etc.) are important in Latinos' use of mental health services. Researchers must address both organizational and cultural barriers to treatment to recruit and retain Latinos in psychotherapy studies.

\section{Recruiting and Retaining Latinos in Psychotherapy Studies}

We present data regarding recruitment and retention of predominantly low-income and less acculturated Latinos from two preventive intervention studies and two treatment studies. These studies have several 
factors in common that aided in recruiting and retaining Latinos in the samples. First, the studies were conducted at San Francisco General Hospital. As mentioned previously, Latinos, particularly less acculturated Latinos, are low users of mental health services. All of these studies were conducted with patients obtained through a medical setting that is situated in and serves the Mission District, a heavily Latino area in San Francisco. All of the studies used bilingual and bicultural staff. All treatments were conducted in Spanish. All of the studies provided services free of charge to the patients. As a result of providing treatment within a Latino setting, in Spanish, and without typical barriers, the studies were accessible to Latinos.

Developing research instruments to be used with Latinos is a controversial field. Although a few studies have been done to develop culturally sensitive Spanish-language instruments or to evaluate the psychometric properties of standard instruments that have been translated into Spanish (for a review, see Marín \& Marín, 1991), many commonly used instruments in psychotherapy research have not been translated into Spanish or normed on Spanish-speaking populations. Our approach to this dilemma has been to carefully translate instruments into Spanish, using back- and forward translation techniques ( Brislin, 1976). We have been able to conduct consensus translations, in which a translation team representing a cross-section of Latino culture back-translates the instrument to ensure appropriate interpretation of the Spanish version for people from different Spanish-speaking countries. We then examine the psychometric properties of the instruments within our research projects.

\section{Preventive Intervention With High-Risk Mother-Infant Dyads}

This research project assessed the effectiveness of infant—parent psychotherapy that focused on improving the infant - mother attachment bond with a group of 100 Latina mothers from Mexico and Central America, all of whom had been residents in the United States for less than 5 years ( Lieberman, Weston, \& Pawl, 1991). Mothers were of low socioeconomic status and not acculturated to the United States. Sixty percent reported high levels of depressive symptoms. All mothers had histories of abuse or neglect. The infants were 12 months at intake and were assessed for quality of attachment ( Ainsworth, Blehar, Waters, \& Wall, 1978 ). Anxiously attached infants were assigned at random to an intervention (infant-parent psychotherapy; Fraiberg, 1979) or to a control group. The groups were assessed for outcomes 1 year later, when the infants were 24 months old.

Mothers and infants were recruited from the well-baby clinics of local hospitals serving a high percentage of Latinos and from neighborhood health clinics. Attrition in this marginalized and highly mobile sample was only $7 \%$. No differential attrition was found between the intervention and the control groups.

The success in retaining the subjects is attributed to a careful individualized approach. Mothers in the control group were telephoned monthly by a female research assistant who was warm, friendly, and interested in their well-being and that of their babies. The research assistant made sure to remember the specific details of each family's situation and to make reference to it, providing a feeling of personal 
rapport and continuity between phone calls. All mothers and babies received birthday cards. Transportation to and from the research site was provided to mothers who needed it. Modest cash reimbursement was given at the end of each assessment. The outcome evaluation was scheduled on the week of the child's second birthday and culminated in a birthday celebration that included cake and a small gift for the child. A videotape of the mother and child interacting, part of the 1-year assessment, was given to the mother at the end of the study as a memento of their participation.

These specific steps were successful because they conveyed the overarching atmosphere of warmth and simpatía that characterized the research team's attitude toward the participants. Far from considering them as research "subjects," the team had a genuine feeling of partnership with the mothers and their babies, and mothers and babies reciprocated in kind.

\section{San Francisco Depression Prevention Research Project}

The San Francisco Depression Prevention Research Project (DPRP) was a randomized controlled

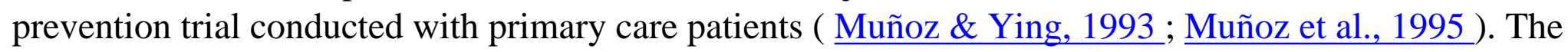
study was conducted in English and Spanish, and recruited participants from primary care clinics at San Francisco General Hospital and the University of California, General Medicine Practice.

In the DPRP, participants were recruited either in face-to-face interactions or through mail. There were major differences in rates of recruitment for these two methods, as well as differences by language group. Mail recruitment yielded acceptance rates of $4.2 \%$ for the English-speaking sample and $4.5 \%$ for the Spanish-speaking sample. Face-tohyphen;face recruitment yielded 24.3\% acceptance for the Englishspeaking sample, and $46.6 \%$ for the Spanish-speaking sample. Once the screening phase was completed, the rate of acceptance for those who were eligible to enter the randomized trial was $94 \%$ for the Englishspeaking and $100 \%$ for the Spanish-speaking. We were able to follow up $93 \%$ of the sample 1 year later.

The aforementioned figures clearly demonstrate that Spanish-speaking individuals are not harder to recruit than English-speaking individuals. These findings support the claim that Latinos' lower rate of utilization of services and lower rate of inclusion into intervention trials is at least partially attributable to not providing culturally and linguistically appropriate staff in these settings. Clearly, bilingual and bicultural staff are able to recruit Latinos into interventions. Among Spanish speakers in the United States, $50 \%$ of those between 18 and 64 years of age, and $62 \%$ of those 65 years old and over speak

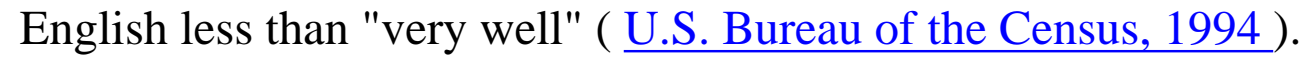

\section{Treatment of Depression in Mothers With Young Children}

Depressed mothers with children less than 3 years old were recruited to participate in a pilot study examining the feasibility of providing group cognitive-behavior therapy combined with infant-parent psychotherapy (principal investigator, F. Azocar). Thirteen mothers were referred to the study, and seven were able to participate. Although referrals to the study were made by both obstetricians and 
pediatricians, we experienced great difficulty in getting the mothers to attend the initial interview. Soon we realized that the mothers were unable to come to our clinic because of child care and transportation limitations. To ensure the recruitment and retention of these mothers, several changes were instituted. The principal investigator made an initial home visit to explain the study and to develop the warm and trusting relationship that allowed us to recruit these women into treatment. The research assistants also interviewed the mothers pre- and posttreatment at their home. To facilitate attendance at treatment sessions, we provided members with free transportation and child care either at home or on site. By providing these services, we were able to achieve an excellent attendance rate by the mothers that averaged 10 of 12 sessions. Through this pilot study, we learned the importance of establishing personal relationships in recruiting young Latinas who may not readily be socialized to put the needs of their family second to get help for themselves. By establishing warm relationships and taking care of family demands (i.e., providing babysitting), we were able to overcome the barriers that prevented these young women from receiving care for their depression.

\section{Treatment of Depression in Disadvantaged Medical Patients}

This study is in the final phases after 5 years of randomizing depressed primary care patients to group cognitive - behavioral therapy or group cognitive-behavioral therapy supplemented with case management (principal investigator, J. Miranda). Patients are referred to the study by primary care physicians in the outpatient departments at San Francisco General Hospital and public health clinics associated with the Hospital. The study was conducted within the Depression Clinic at San Francisco General Hospital, a specialty clinic developed to treat depression in medical patients referred within the public care medical system.

In this study, we successfully recruited low income and minority patients into treatment by being intimately tied with the medical clinics referring patients to us. The principal investigator of the study was also a faculty member in the Department of Medicine at San Francisco General Hospital and taught primary care residents about psychiatric aspects of medical illness. In addition, she spent one half day per week consulting in the general medical clinic, allowing her to develop strong relationships with the referral physicians. She was able to teach primary care physicians to identify and refer depressed patients; part of this training involved cross-cultural understandings of psychiatric presentations. The population of patients seen in the medical clinics is $23 \%$ Latino; the sample of patients referred by their physicians for the study was $24.9 \%$ Latino. As a result of cross-cultural training and awareness on the part of the physicians, Latinos were as readily identified as depressed as were non-Latino medical patients.

After Latinos were referred to this study, we were able to successfully recruit them into treatment. In fact, $17.6 \%$ of referred non-Latinos were entered into the trial; whereas, $32.9 \%$ of referred Latinos entered the study. The Latinos in our sample tended to have excellent relationships with their primary care providers at San Francisco General Hospital; many of the providers were bilingual, bicultural, or both. The patients were willing to participate in our study on the recommendation of their physicians. Once in the study, our bilingual-bicultural staff were able to maintain Latino patients in treatment at the 
same rate as our non-Latino sample.

We were successful at retaining Latinos in our sample. However, we did find it necessary to extend extra efforts to maintain many of our Latino patients in treatment. For example, we reduced our original high dropout rate by calling Latinos the day before each therapy session. We found it necessary to make these reminder telephone calls during the first 2 months of treatment with Latinos. We also maintained Latinos in therapy by responding to their concerns that the treatment was too environmentally cold. We began providing coffee and cold drinks during all treatment sessions. In addition, we allowed patients to bring food to their final group sessions to share among group patients. These simple adjustments, which were not necessary in the English-language groups in our study, helped to maintain Latinos in treatment.

\section{Conclusion}

The major conclusion that we draw from our review of the literature and our own experience is that Latinos can be recruited and retained in treatment outcome studies. To do this successfully, bilingual and bicultural staff should be part of a research team. In addition, Latinos should be recruited in settings other than mental health clinics. Also, Latinos may need to overcome barriers, such as child care responsibilities and lack of transportation, to attend treatment. Providing these services increases the rate of participation in studies. Finally, forming a respectful, warm, and personal relationship with Latinos is critical to their successful recruitment and retention in psychotherapy outcome studies. Systematic, even if unintentional, exclusion of a group comprising over $10 \%$ of the U.S. population from the psychotherapy literature is questionable on both scientific and ethical grounds.

\section{References}

Acosta, F. X. \& Sheehan, J. G. (1976a). Preferences toward Mexican-American and Anglo-American psychotherapists.( Journal of Consulting and Clinical Psychology, 44, 272-279.)

Acosta, F. X. \& Sheehan, J. G. (1976b). Psychotherapist ethnicity and expertise as determinants of selfdisclosure.( Spanish-Speaking Mental Health Research Center Monograph Series, 3, 51-59.)

Ainsworth, M. D. S., Blehar, M., Waters, E. \& Wall, S. (1978). Patterns of attachment: A psychological study of the Strange Situation. (Hillsdale, NJ: Erlbaum)

Barrera, M. (1978). Mexican-American mental health service utilization: A critical examination of some proposed variables.( Community Mental Health Journal, 14, 35-45.)

Bernal, G. \& Gutierrez, M. (1988). Cubans.(In L. Comas-Diaz \& E. H. G. Erna (Ed.), Clinical guidelines in cross-cultural mental health. Wiley series in general and clinical psychiatry (pp. 233-261). New York: Wiley.)

Blackburn, I. M., Bishop, S., Glen, A. I. M., Whalley, L. J. \& Christie, J. E. (1981). The efficacy of cognitive therapy in depression: A treatment trial using cognitive therapy and pharmacotherapy, each alone and in combination.( British Journal of Psychiatry, 139, 181-189.)

Brislin, R. (1976). Comparative research methodology: Cross-cultural studies.( International Journal of Psychology, 11, 215-229.) 
Comas-Diaz, L. (1981a). Effects of cognitive and behavioral groups treatment on the depressive symptomatology of Puerto Rican women.( Journal of Consulting and Clinical Psychology, 49, 627-632.)

Comas-Diaz, L. (1981b). Puerto Rican expiritismo and psychotherapy.( American Journal of Orthopsychiatry, 51, 636-645.)

Comas-Diaz, L. \& Duncan, J. W. (1985). The cultural context: A factor in assertiveness training with mainland Puerto Rican women.( Psychology of Women Quarterly, 9, 463-475.)

Costantino, G., Malgady, R. G. \& Rogler, L. H. (1986). Cuento therapy: A culturally sensitive modality for Puerto Rican children.( Journal of Consulting and Clinical Psychology, 54, 639-645.)

De La Cancela, V. \& Zavala, I. (1983). An analysis of culturalism in Latino mental health: Folk medicine as a case in point.( Hispanic Journal of Behavioral Sciences, 5, 251-274.)

DiMascio, A., Weissman, M. M., Prusoff, B. A., Neu, C., Zwilling, M. \& Klerman, G. L. (1979). Differential symptom reduction by drugs and psychotherapy in acute depression.( Archives of General Psychiatry, 36, 1450-1456.)

Edgerton, R., Karno, M. \& Fernandez, I. (1970). Curanderismo in the metropolis: The diminished role of folk psychiatry among Los Angeles Mexican-Americans.( American Journal of Psychotherapy, 24, 124-134.)

Elkin, I., Shea, M. T., Watkins, J. T., Imber, S. D., Sotsky, S. M., Collins, J. E., Glass, D. R., Pilkonis, P. A., Leber, W. R., Docherty, J. P., Fiester, S. J. \& Parloff, M. B. (1989). National Institute of Mental Health Treatment of Depression Collaborative Research Program: General effectiveness of treatments.( Archives of General Psychiatry, 46, 971-982.)

Figueroa-Torres, J. \& Pearson, R. E. (1979). Effects of structured learning therapy upon self-control of aggressive Puerto Rican fathers.( Hispanic Journal of Behavioral Sciences, 1, 345-354.)

Fraiberg, S. (1979). Clinical studies in infant mental health. (Basic Books)

Guarnaccia, P. J., Canino, G., Rubio-Stipec, M. \& Bravo, M. (1993). The prevalence of ataques de nervios in the Puerto Rico Disaster Study: The role of culture in psychiatric epidemiology.( Journal of Nervous and Mental Disease, 181, 157-165.)

Healey, J. F. (1995). Race, ethnicity, gender, and class: The sociology of group conflict and change. (Thousand Oaks, CA: Pine Forge Press)

Hersen, M., Bellack, A. S., Himmelhoch, J. M. \& Thase, M. E. (1984). Effects of social skill training, amitriptyline, and psychotherapy in unipolar depressed women.( Behavior Therapy, 15, 21-40.) Hollon, S. D., DeRubeis, R. J., Evans, M. D., Wiemer, M. J., Garvey, M. J., Grove, W. M. \& Tuason, V. B. (1992). Cognitive therapy and pharmacotherapy for depression: Singly and in combination.( Archives of General Psychiatry, 46, 774-781.)

Hough, R. L., Landsverk, J. A., Karno, M., Burnam, M. A., Timbers, D. M., Escobar, J. I. \& Regier, D. A. (1987). Utilization of health and mental health services by Los Angeles Mexican Americans and nonHispanic Whites.( Archives of General Psychiatry, 44, 702-709.)

Karno, M. \& Edgerton, R. B. (1969). Perception of mental illness in a Mexican American community.( Archives of General Psychiatry, 20, 233-238.)

Karno, M., Ross, R. \& Caper, R. (1969). Mental health roles of physicians in a Mexican-American community.( Community Mental Health Journal, 5, 62-69.)

Keefe, S. E., Padilla, A. M. \& Carlos, M. L. (1978). In J.(M. Casas \& S. E. Keefe (Eds.), Family and mental health in the Mexican American community (Monograph No. 7). Los Angeles: University of 
California, Spanish-Speaking Mental Health Research Center.)

Kessler, R. C., McGonagle, K. A., Zhao, S., Nelson, C. B., Hughes, M., Eshlemman, S., Wittchen, H. \& Kendler, K. S. (1994). Lifetime and 12-month prevalence of $D S M-I I I-R$ psychiatric disorders in the United States.( Archives of General Psychiatry, 51, 8-19.)

Leaf, P., Bruce, M., Tischler, G. \& Holzer, C. (1987). The relationship between demographic factors and attitudes toward mental health services.( Journal of Community Psychology, 15, 275-284.)

Lieberman, A. F., Weston, D. R. \& Pawl, J. H. (1991). Preventive intervention and outcome with anxiously attached dyads.( Child Development, 62, 199-209.)

Loo, C., Tong, B. \& True, R. (1989). A bitter bean: Mental health status and attitudes in Chinatown.( Journal of Community Psychology, 17, 283-296.)

Malgady, R. G., Rogler, L. H. \& Costantino, G. (1990). Hero/heroine modeling for Puerto Rican adolescents: A preventive mental health intervention.( Journal of Consulting and Clinical Psychology, 58, 469-474.)

Marin, B., Marin, G., Padilla, A. \& de la Rocha, C. (1983). Utilization of traditional and non-traditional sources of health care among Hispanics.( Hispanic Journal of Behavioral Sciences, 5, 65-80.)

Marín, G. \& Marín, B. (1991). Research with Hispanic populations (Vol.(23). Newbury Park, CA: Sage) Meinhardt, K. \& Vega, W. (1987). A method for estimating underutilization of mental health services by ethnic groups.( Hospital and Community Psychiatry, 38, 1186-1190.)

Moscicki, E. K., Locke, B. Z., Rae, D. S. \& Boyd, J. H. (1989). Depressive symptoms among Mexican Americans: The Hispanic Health and Nutritional Examination Survey.( American Journal of Epidemiology, 130, 348-360.)

Muñoz, R. F. \& Ying, Y. (1993). The prevention of depression: Research and practice. (Baltimore: Johns Hopkins University Press)

Muñoz, R. F., Ying, Y. W., Bernal, G., Pérez-Stable, E. J., Sorensen, J. L., Hargreaves, W. A., Miranda, J. \& Miller, L. S. (1995). Prevention of depression with primary care patients: A randomized controlled trial.( American Journal of Community Psychology, 23, 199-213.)

Murphy, G. E., Simons, A. D., Wetzel, R. D. \& Lustman, P. J. (1984). Cognitive therapy and pharmacotherapy.( Archives of General Psychiatry, 41, 33-41.)

Narrow, W. E., Rae, D. S., Moscicki, E. K., Locke, B. Z. \& Regier, D. A. (1990). Depression among Cuban Americans: The Hispanic Health and Nutrition Examination Survey.( Social Psychiatry and Psychiatric Epidemiology, 25, 260-268.)

Navarro, A. M. (1993). Efectividad de las psicoterapias con Latinos en los Estados Unidos: Una revision meta-analitica [The effectiveness of psychotherapy with Latinos in the United States: A meta-analytic review].( Interamerican Journal of Psychology, 27, 131-146.)

Norquist, G. \& Wells, K. (1991a). How do HMOs reduce outpatient mental health care costs? American Journal of Psychiatry, 148, 96-101.( Norquist G. Wells K. 1991b Archives of General Psychiatry 48, 475-478.)

Rogler, L. H., Cortes, D. E. \& Malgady, R. G. (1991). Acculturation and mental health status among Hispanics: Convergence and new directions for research.( American Psychologist 46, 585-597.)

Rush, A. J., Beck, A. T., Kovacs, M. \& Hollon, S. (1977). Comparative efficacy of cognitive therapy and pharmacotherapy in the treatment of depressed outpatients.( Cognitive Therapy and Research 1, 17-38.) Snowden, L. R. \& Cheung, F. K. (1990). Use of inpatient mental health services by members of ethnic minority groups.( American Psychologist 45, 347-355.) 
Sue, S., Fujino, D. C., Hu, L.-T., Takeuchi, D. T. \& Zane, N. W. S. (1991). Community mental health services for ethnic minority groups: A test of the cultural responsiveness hypothesis.( Journal of Consulting and Clinical Psychology 59, 533-540.)

Szapocznik, J., Santisteban, D., Rio, A., Perez-Vidal, A., Kurtines, W. \& Hervis, O. (1986). Bicultural effectiveness training (BET): An intervention modality for families experiencing intergenerational/intercultural conflict.( Hispanic Journal of Behavioral Sciences 8, 303-330.)

Takeuchi, D., Leaf, P. \& Kuo, H. S. (1988). Ethnic differences in the perception of barriers to helpseeking.( Social Psychiatry \& Psychiatry Epidemiology, 23, 273-280.)

U.S. Bureau of the Census. Statistical abstracts of the United States (110th ed.). Washington DC: U.S. Government Printing Office.

U.S. Bureau of the Census. Statistical abstracts of the United States (114th ed.). Washington, DC: U.S. Government Printing Office.

Vernon, S. W. \& Roberts, R. E. (1982). Prevalence of treated and untreated psychiatric disorders in three ethnic groups.( Social Science and Medicine, 16, 1575-1582.)

Wells, K. B., Hough, R. L., Golding, J. M., Burnam, M. A. \& Karno, M. (1987). Which Mexican Americans underutilize health services? American Journal of Psychiatry, 144, 918—922. 